\section{UN CLÁSICO DE LA SOCIOLOGÍA (URBANA) FRANCESA}

\author{
Paul Pasquali ${ }^{1}$
}

Traducción de Luis Campos M. y Malena

Bastías S.

Jean-Claude Chamboredon es el autor de una obra singular, poco conocida en América Latina, pero significativa para muchos investigadores francófonos en ciencias sociales, como lo demuestran los homenajes que le fueron rendidos luego de su muerte en 2020, a la edad de 82 años ${ }^{2}$. Si su nombre es usualmente asociado a los de Pierre Bourdieu y Jean-Claude Passeron, con quienes publicó en 1968 El oficio de sociólogo (Bourdieu, Chamboredon y Passeron, 2002), la posteridad ha conservado de su obra

1 Investigador del Centre National de la Recherche Scientifique (CNRS). Integrante del Centre Universitaire de Recherche sur l'Action Publique et le Politique, Amiens, Francia. Correo electrónico: pasqualipaul@gmail.com

\section{A CLASSIC OF FRENCH (URBAN) SOCIOLOGY}

Paul Pasquali

dos artículos que se han convertido en clásicos y que siguen siendo ampliamente citados en Francia, ambos publicados en la Revue Française de Sociologie. Se trata de "Proximidad espacial y distancia social", publicado en 1970 junto a Madeleine Lemaire, una investigadora asistente que entonces se desempeñaba como trabajadora temporal en el Centro de Sociología Europea dirigido por Bourdieu, y "Delincuencia juvenil, ensayo de construcción de objeto", publicado el año siguiente $y$ firmado únicamente

2 Véase, por ejemplo, el homenaje de la EHESS y de diversos investigadores marcados por su trabajo como Jean-Louis Fabiani, François Héran, Pierre-Michel Menger, Jacques Revel o Florence Weber (Centre Norbert Elias, s.f.). Véase también Fabiani, 2020. 
por Chamboredon (1971; Chamboredon y Lemaire, 1970). Estos dos artículos se centran en fenómenos que los sociólogos franceses de los años cincuenta y sesenta dejaban en gran medida a los periodistas, trabajadores sociales y ensayistas de todo tipo. La sociedad francesa se encontraba entonces en un período de importantes cambios en el que se construían masivamente grandes conjuntos de vivienda, así como "ciudades nuevas" en los suburbios, con el fin de reubicar a las familias expulsadas de los centros urbanos en proceso de renovación, y de absorber las olas migratorias, cada vez más importantes luego del inicio del proceso de descolonización.

Negándose a confundir los problemas sociales y los problemas sociológicos, de acuerdo con la distinción que se encuentra en el corazón de El oficio de sociólogo, Chamboredon y Lemaire eligieron un (sugerente) título que resume bien su perspectiva científica. Rechazando todas las profecías de los años sesenta, tanto aquellas planteadas por los poderes públicos, como las de los discursos humanistas que anunciaban la "reconciliación" de las clases sociales mediante su agrupación en grandes conjuntos de vivienda que simbolizaban el progreso social y la modernidad urbana, los autores mostraron que la convivencia de poblaciones socialmente diferenciadas, lejos de provocar un acercamiento de clases, acentúa las tensiones entre los grupos involucrados. Estas tensiones se cristalizan, principalmente, en juicios sobre el comportamiento de los jóvenes -en particular los de origen obrero, que son más numerosos y más visibles en los espacios públicos- y sobre el estilo de educación familiar que se imparte a los niños, en los que se plasma el estatus y prestigio al que aspiran sus padres.

Tal resultado contradecía los hallazgos de la investigación realizada por separado por tres prominentes investigadores mencionados explícitamente en el artículo. Se trataba, en primer lugar, de Paul-Henry Chombart de Lauwe, autor de la primera gran investigación en sociología urbana realizada en Francia (Chombart de Lauwe, 1952). Según él, los grandes conjuntos de vivienda allanaban el camino para una sociedad libre de conflictos de clases gracias a su acercamiento espacial. La segunda referencia apuntaba a Henri Lefebvre, filósofo marxista para quien los grandes conjuntos encarnaban la promesa de liberación de la alienación del proletariado, mediante la regeneración de los vínculos comunitarios (Lefebvre, 1968). Finalmente, el tercer aludido era Paul Clerc, demógrafo, autor de un vasto estudio estadístico sobre las actitudes y aspiraciones de los habitantes de los grandes conjuntos habitacionales (Clerc, 1967). Según él, estos "nuevos barrios", tanto por su morfología social como por las actitudes de sus habitantes, no diferían del promedio de la población general. En consecuencia, los "problemas sociales" que allí se presentaban no tenían nada de anormal. 
Chamboredon y Lemaire disentían de estas tres variantes del optimismo que prevalecía en aquel entonces entre muchos intelectuales y líderes políticos acerca de los grandes conjuntos habitacionales (para mayores antecedentes ver Pasquali, 2012). Su trabajo mostró que esta nueva forma de vivienda colectiva presentaba una morfología social muy particular, mezclando poblaciones heterogéneas que no surgían ni se encontraban juntas de forma azarosa, sino que eran producidas por mecanismos sociales. Y era precisamente esta heterogeneidad la fuente de los "problemas" que surgían en los grandes conjuntos habitacionales o en su entorno inmediato, especialmente en las zonas residenciales cercanas a ellos. Para llegar a esta conclusión, fue necesario desplazar la mirada de los debates normativos acerca de las políticas de vivienda y construir un razonamiento crítico a partir de una profunda investigación de terreno y de estadísticas originales, más finas que las utilizadas usualmente en esta temática, para así hacer posible el análisis de fenómenos a escala de la comuna, de cada barrio y de cada edificio.

El punto de partida de este artículo es una investigaciónexploratoria, realizada por Lemaire en 1966 en Creil, una pequeña ciudad en auge al norte de París, entre los habitantes de un gran conjunto habitacional recientemente creado. En su trabajo de campo, la socióloga comprendió rápidamente que, de existir un "malestar" en los grandes conjuntos de vivienda, tal como lo sugerían los debates recurrentes en ese momento acerca de la "sarcellite", este remitía a que la proximidad de las clases desfavorecidas perturba a las clases acomodadas, provocando entre los habitantes de ambas, un sentimiento de nostalgia o un deseo de segregación social. Es esta constatación la que luego se convertirá en el hilo conductor de la investigación que ella y Chamboredon realizarán entre 1967 y 1969 en el gran complejo de Antony-Massy, ubicado en los suburbios del sur de París. En ese momento, este gran conjunto habitacional era el segundo más poblado de Francia, después del de Sarcelles, ubicado en la periferia norte de París. Pero la elección de este terreno obedeció fundamentalmente a que la investigación podría llevarse a cabo en condiciones especialmente favorables. En primer lugar, Bourdieu vivía en Antony, y sus buenas relaciones con el alcalde facilitaron enormemente las cosas a lo largo de toda la investigación. Además, existía una gran preocupación entre los administradores de clubes juveniles y los representantes locales respecto de la baja dotación de infraestructuras culturales en el gran conjunto habitacional. Para remediar esta situación, Bourdieu fue contactado por un miembro del equipo

3 Este término, muy empleado a partir de la década de los sesenta, alude a la supuesta enfermedad propia de los grandes conjuntos habitacionales: una suerte de depresión o angustia que era muy frecuente entre sus habitantes, como resultado de la enorme concentración de viviendas sin otro tipo de equipamientos (culturales, deportivos, etcétera). Se deriva de la localidad en que se emplazaba el conjunto habitacional más grande de Francia, Sarcelles, en los suburbios del norte de París [NdelosT] 
municipal, presidente de un club juvenil. Así, sin ser el resultado de un encargo público, esta encuesta respondió principalmente a una demanda social. Pero esta última fue traducida en los términos y de acuerdo con la lógica específica de los problemas científicos propugnada por ambos sociólogos. En ese tiempo Bourdieu pretendía hacer de Antony una especie de "laboratorio sociológico" donde se realizaría una serie de trabajos monográficos acumulativos sobre diversos temas (educación, cultura, hábitat, profesiones intelectuales, delincuencia, medicina, etc.), con el fin de crear un "Chicago a la francesa" para el que Chamboredon, junto con Lemaire, sentarían las bases. Si este vasto proyecto no tuvo continuidad, encontramos en esta investigación algunas huellas implícitas, pero sobre todo las encontramos en las referencias explícitas a la "escuela de Chicago" que se hacen en el artículo sobre la delincuencia juvenil (ver a este respecto Pasquali, 2018).

Contrariamente a la tendencia frecuente que reduce Chicago al fieldwork y a unos vagos conceptos interaccionistas, Chamboredon y Lemaire nunca defienden en su investigación una postura enteramente "cualitativista", lo que les hubiera llevado a preferir las observaciones in situ por sobre las demostraciones estadísticas, áridas y laboriosas. Esta originalidad merece atención, no por una suerte de elogio del método, sino para insistir en un aspecto crucial de este tipo de sociología que aún no había sido calificada como "crítica" en los años 19601970, período en el que la figura de Bourdieu todavía no había eclipsado por completo a la mayoría de sus colaboradores, comenzando por Chamboredon. Más allá de su temática explícita, que inicialmente interesará a los especialistas en estudios urbanos, "Proximidad espacial y distancia social" es sobre todo una contribución a una sociología general que integra los aportes de Durkheim, Marx y Weber. Esta ambición estaba lejos de ser obvia hace 50 o 60 años. El advenimiento de lo que algunos autores han llamado un "giro etnográfico" en la sociología no quita nada de actualidad a esta apuesta teórica, al contrario: asociando sistemáticamente teoría y empirismo, estadística y etnografía, pero también yendo más allá del antagonismo académico entre objetivismo y subjetivismo, este artículo ejemplifica todo el interés de una sociología total, es decir, una que "equipa" el campo con teoría y estadística.

Todo sociólogo sabe lo que se puede ganar con un enfoque de investigación generalista y acumulativo, que permita evitar los impasses que encuentran tantos especialistas que confinan su tema dentro del estrecho perímetro de su jerga y de sus preguntas, sin reflexionar acerca de lo que tal tecnicismo pueda tener de "científico". Lo mismo ocurre con los amantes de los grandes modelos o de las teorías sofisticadas, para quienes la relación con el campo es puramente instrumental y sólo sirve para "probar" intuiciones mal controladas y que, 
en general, sólo reproducen el sentido común. Ahora bien, si 50 años después, seguimos hablando de este artículo de Chamboredon y Lemaire, ¿no es, precisamente, porque ellos lograron evitar estos escollos? Por cierto, la influencia de una investigación está ligada a su originalidad y su rigor, pero quizás también (y sobre todo) a su capacidad para desafiar la "mezcla de géneros" cuando esta consiste en amputar a la investigación una de sus piernas, ya sea la empírica o la teórica.

Sin embargo, no hay ninguna razón para fetichizar un artículo escrito en una época que en gran parte ha desaparecido. Los grandes conjuntos de los que aquí hablan Chamboredon y Lemaire perdieron rápidamente la mezcla social que podían presentar en Francia en los años sesenta y setenta. Transformaciones estructurales como la creciente pauperización de las clases populares, separadas paulatinamente del grupo obrero, a su vez mucho menos central y combativo que antes, el éxodo de las clases medias que prefieren vivir en áreas residenciales o en centros urbanos rehabilitados, el avance de la segregación en todo el territorio o, incluso, la homogeneización étnica de los conjuntos de vivienda social, cambió la situación a partir de los años 1980-1990. Los conflictos entre clases o fracciones de clase que antes predominaban han sido reemplazados progresivamente por luchas internas dentro de la misma clase o fracción de clase, por conflictos y tensiones entre individuos separados por su religión, su color de piel o, más a menudo aún, por su relación con el futuro, la que se encuentra fuertemente ligada a diferencias en las trayectorias educativas y/o familiares. A pesar de todo, las conclusiones de Chamboredon y Lemaire siguen siendo válidas, a condición de que se tengan en cuenta las transformaciones sociales, económicas y urbanísticas que se han producido desde 1970.

Este artículo ha tenido una acogida favorable, pero variada según la época. Entre los sociólogos urbanos fue inmediatamente leído y reconocido, pero muy poco discutido. Manuel Castells lo ha citado varias veces, pero sin detenerse demasiado en él. Los partidarios de una "ecología urbana" inspirada en Chicago se han mostrado más entusiastas, como Yves Grafmeyer e Isaac Joseph (1984). El texto fue criticado en los años 1980-1990 por sociólogos urbanos reunidos en torno a Michel Anselme y Michel Peraldi, quienes llamaban a dar un mayor énfasis a las poblaciones inmigrantes y a los efectos de las trayectorias migratorias en el estudio de las sustituciones de población y las lógicas institucionales de asignación de vivienda, tanto en aquellas efectuadas por agencias sociales, como por el Estado. Más recientemente, investigaciones sobre las áreas residenciales adyacentes a los grandes conjuntos habitacionales han mostrado el interés de incluir en el análisis a los grupos ubicados en las fronteras de las clases populares y las clases medias, los llamados "medio-emergentes" 
(Cartier, Coutant, Masclet y Siblot, 2008), marcados por su ascenso social y su miedo a la degradación y el desclasamiento, y que a veces son tentados por un discurso racista que alimenta constantemente los problemas cotidianos en los conjuntos habitacionales aledaños.

Más allá de la sociología, este artículo ha tenido una gran influencia entre geógrafos e historiadores urbanos. Marcel Roncayolo, a menudo lo ha clasificado entre los textos esenciales, al igual que Thierry Paquot. Pero es sin duda entre los profesionales del urbanismo donde el artículo ha servido y sigue sirviendo de base para la reflexión. A partir de los años 1990-2000, las políticas urbanas en Francia tuvieron que abandonar una visión ingenua de una mezcla social totalmente condicionada por el espacio y por las decisiones estatales, debido a que todos los indicadores mostraban una segregación urbana creciente y la formación de "pequeños guetos". El artículo de Chamboredon y Lemaire, que no buscaba en modo alguno dar una "solución" a estos problemas, sino más bien iluminar la reflexión, fue y sigue siendo un antídoto contra el voluntarismo institucional. La crítica mordaz que planteaba hace cinco décadas no ha envejecido ni un ápice, especialmente para todos aquellos que hoy se interrogan, ya sea como sociólogos o como lectores de sociología, sobre las múltiples dimensiones de la segregación urbana y su papel en lo que Robert Castel (2007), muy influido por el texto de Chamboredon y Lemaire, ha denominado la "discriminación negativa".

\section{Referencias bibliográficas}

Bourdieu, P., Chamboredon, J.-C., y Passeron, J.-C. (2002). El oficio de sociólogo. Buenos Aires: Siglo XXI.

Cartier, M., Coutant, I., Masclet, 0., y Siblot, Y. (2008). La France des «petits moyens». Enquête sur la banlieue pavillonnaire. París: La Découverte.

Castel, R. (2007). La discrimination négative: citoyens ou indigènes? París: Seuil.

Centre Norbert Elias. (s.f.). Hommage à Jean-Claude Chamboredon. EHESS

https://www.ehess.fr/fr/hommage/ hommage-jean-claude-chamboredon

Chamboredon, J.-C. (1971). La délinquance juvénile, essai de construction d'objet. Revue Française de Sociologie, 12(3), 335-377. https://doi.org/10.2307/3320235

Chamboredon, J.-C. y Lemaire, M. (1970). Proximité spatiale et distance sociale. Les grands ensembles et leur peuplement. Revue Française de Sociologie, 11(1), 3-33.

https://doi.org/10.2307/3320131

Chombart de Lauwe, P.-H. (1952). Paris et l'agglomération parisienne. París: Puf.

Clerc, P. (1967). Grands ensembles, banlieues nouvelles. Enquête démographique et psycho-sociologique. París: Puf. 
Fabiani, J. (2020). In memoriam: Jean-Claude Chamboredon (1938-2020). Revue Française de Sociologie, 61(1), 7-12.

https://doi.org/10.3917/rfs.611.0007

Grafmeyer, Y. y Joseph, I. (1984). L'école de Chicago. Naissance de lécologie urbaine. Paris: Aubier.

Lefebvre, H. (1968). Le droit à la ville. París: Anthropos.

Pasquali, P. (2012). Deux sociologues en banlieue: L'enquête sur les grands ensembles de Jean-Claude Chamboredon et Madeleine Lemaire (1966-1970). Genèses, 87(2), 113-135. https://doi.org/10.3917/gen.087.0113

Pasquali, P. (2018). Une «école de Chicago» en banlieue parisienne? Jean-Claude Chamboredon et la délinquance juvénile, de l'article à l'enquête. En G. Laferté, P. Pasquali, y N. Renahy, (Dirs.), Le laboratoire des sciences sociales. Histoires d'enquêtes et revisites (pp. 235-291). París: Raisons d'Agir. 


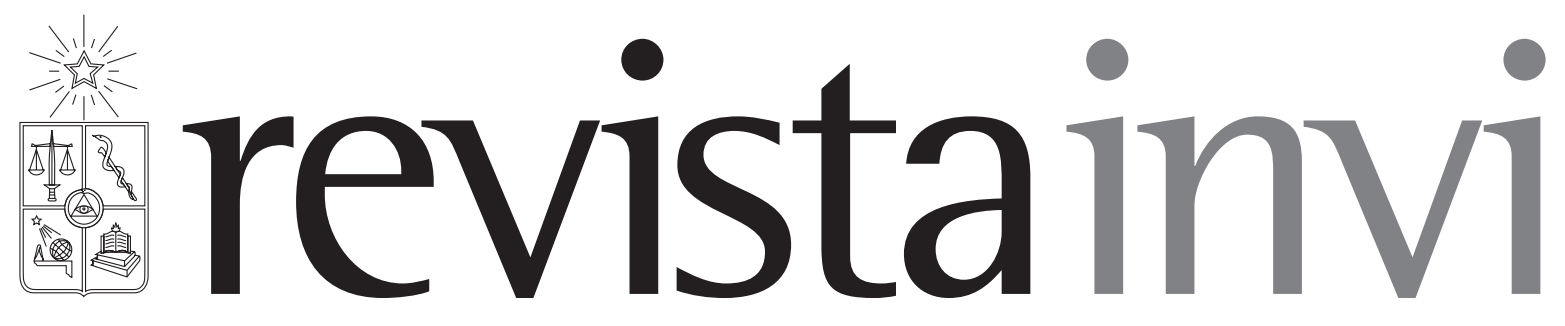

Revista INVI es una publicación periódica, editada por el Instituto de la Vivienda de la Facultad de Arquitectura y Urbanismo de la Universidad de Chile, creada en 1986 con el nombre de Boletín INVI. Es una revista académica con cobertura internacional que difunde los avances en el conocimiento sobre la vivienda, el hábitat residencial, los modos de vida y los estudios territoriales. Revista INVI publica contribuciones originales en español, inglés y portugués, privilegiando aquellas que proponen enfoques inter y multidisciplinares y que son resultado de investigaciones con financiamiento y patrocinio institucional. Se busca, con ello, contribuir al desarrollo del conocimiento científico sobre la vivienda, el hábitat y el territorio y aportar al debate público con publicaciones del más alto nivel académico.

Director: Dr. Ricardo Tapia Zarricueta, Universidad de Chile, Chile.

Editor: Dr. Luis Campos Medina, Universidad de Chile, Chile.

Editor asistente: Dr. Walter Imilan, Universidad de Chile, Chile.

Coeditora: Srta. Sandra Rivera, Universidad de Chile, Chile.

\section{COMITÉ EDITORIAL:}

Dr. Victor Delgadillo, Universidad Autónoma de la Ciudad de México, México.

Dra. María Mercedes Di Virgilio, CONICET/ IIGG, Universidad de Buenos Aires, Argentina.

Dra. Irene Molina, Uppsala Universitet, Suecia.

Dr. Gonzalo Lautaro Ojeda Ledesma, Universidad de Valparaíso, Chile.

Dra. Suzana Pasternak, Universidade de São Paulo, Brasil.

Dr. Javier Ruiz Sánchez, Universidad Politécnica de Madrid, España.

Dra. Elke Schlack Fuhrmann, Pontificia Universidad Católica de Chile, Chile.

Dr. Carlos Alberto Torres Tovar, Universidad Nacional de Colombia, Colombia.

Sitio web: http://www.revistainvi.uchile.cl/

Correo electrónico: revistainvi@uchilefau.cl

Licencia de este artículo: Creative Commons Atribución-Compartirlgual 4.0 Internacional (CC BY-SA 4.0) 